ceived, though they are scarcely supported either by facts or arguments. He appears to maintain, that calculi are not formed by any chemical deposition or crystallisation of salts from the urine, but that their particles are secreted in a solid form, and that not only by the kidneys, but even by the mu. cous coat of the bladder, for he speaks at page 9 of Dr. Marcet's being misled by his too exclusive attention to chemical affinities when he stated, that " independent of any specific agency of the urinary organs themselves, calculi are liable to form in any of the cavities to which the urine has access," and subsequently at page 27 observes,

"That the phosphates may be secreted by the vesical arteries, and thus have their orioin ab initio in the bladder, cannot be aoubted."

His reasons for this latter statement he does not mention, and we know of no circumstance which might render it even probable, except that of calculi being found in the ducts of the prostate, where they could scarcely be deposited by the urine; this, however, is any thing but indisputable evidence; and it is far more probable, that in every case calculi are formed by the gradual deposition of salts from the urine.

With regard to the style, it has the fault too common in second -rate medical works of the present day, of neither being sufficiently concise, nor confincd to the proper subjects of the work. How misplaced, for instance, is such a sentence as the following, "And we are well aware, that, from the earliest dawn of memory, the notoriety of Ireland's butter-milk and potatoes has not been limited to the mountains and bogs between Cape Clear and the Giant's Causeway!'- - not to speak of the absurdity of such an expression as "the earliest dawn of memory," which can here have no definite meaning whatever. The author appears to possess some talent for observation, though he would certainly have acted much more wisely in delaying the publication of his book until be had collected more information, arranged his arguments better, and inquiled further into the truth of those facts which he states with so much positive as. sertion.

\section{EXCISION OF THE UPPER JAW- BONE.}

\section{By John Lizars, Esq.,}

\section{Lecturer on Anatomy, Edinburgh.}

As the operation for the removal of the upper jaw-bone is still in its infancy, and as " truth scarcely ever yet carried it by vote anywhere at its first appearance, new opinions being always suspected, and usually opposed without any other reason but because they are not already common," I have sent you an account of it, "for trial? and examination" must give it publicity.

In 1826, I proposed in Part ix. p. 58 of my anatomical work, the entire removal of the superior maxillary bone, when its cavity is affected with those sarcomatous tumours which become so malignant if $l \in f t$ to them. selves, and so fatal if attempted to be removed by trejhining the anterior wall of the antrum. Accoráingly, in December, 1827, I attempted to remove this bone for a medullary sarcomatous tumour of the antrum, after securing the common carotid artery of the affected side, but I was prevented by the hamorrhagic disposition of the gum and palate, my patient having lost, in a few seconds, upwards of two pounds of blood, which welled out at every incision, as if there had been aneurism by anastomosis. The man, who was a strong athletic miner or collier, survived this attempt seventeen months, lingering out a most loathsome existence, and suffering great agony for weeks prior to his decease. His face became frightfully distorted, the eyes separated from each other to a great distance, starting from their sockets, and being quite amaurotic; the cornea of the right eye had ruptured from the pressure, and the iris protruded; the nose got flattened or concenled; the mouth was wide open; a sinuous ulcer appeared on the forehead; and the glands on each side of the neck, especially those of the right, were enlarged, indurated, and ulcerated. I have preserved a cast of the above appearances in my museum. The trmour, purely a medullary sarcoma, was highly vascular, occupied both antra, having destroyed tive septum narium, filled the whole of the nares, and extended up into the brain, having absorbed the orbital plates of the frontal bone, the whole of the ethmoid, and the body of the sphenoid. It was with difficulty distinguished from the brain. My second case is detailed in a contemporary medical journal, with, however, an egregious mistale, the reporter having stated, that the operation was performed " three weeks subsequent to her labouring under general dropsy ;" whereas the period was no less than as many years.

The third operation, to which I now call 
your attention, was performed by me on the 10th of January last, in the case of a healthy woman fifty-five years of age, who was placed under my care by Dr. Simmons of Dumfries. Eight months before this period she began to feel pain in her right temple and eye, the latter of which soon protruded in a slight degree, and immediately afterwards the cheek; a fetid discharge began to flow from the nostril of the affected side, and a tumefaction of the hard palate to appear. When she arrived in Edinburgh, there was, on the right side, a distinct bulging downwards of the bard palate, an evident protrusion of the superior maxillary bone forwards to the face, and upwards to the eye, which organ had a weeping, inflamed, and swollen appearance; there was also a small, round, firm tumour in the nostril, from which issued a sanious discharge.

Having prescribed a cathartic the day before, I operated as follows: I placed $\mathrm{my}$ patient on a table, secured on the right side the trunk common to the temporal and internal maxillary arteries, immediately below the posterior belly of the digastric muscle, slit up the right nostril close to the mesial septum, likewise the ulper lip at the labial fossa, and the cheek from the angle of the mouth to the masseter muscle. I next divested the bone of its soft coverings, at the points where the saw was to be applied, by dividing the mucous membrane which invests the floor of the naris, also the gum from the naris to the palate, the palate backwards to the velum, and across to the dens sapientiæ, carefully preserving the ad. besion of the velum to the palatine plate of the os palati; after which I dissected up the flap of the cheek from the superior maxillary bone towards its nasal process, and to where it forms the flcor of the orbit, also outwards towards the cheek-bone, and around to its bulbous process.

The superior maxillary bone being thus divested of its soft coverings, or cleared of these where it was intended to be removed, I applied the saw at the following difierent places, namely, the front of the bone between naris and mouth, or at the side of the mystachial suture; the palatine plate backwards from this, parallel with the longitudinal palatine suture, to near where the transverse palatine suture exists; across the same palatine plate towards the bulbous process; upwards between the bulbous process and the pterygoid processes of the sphenoid bone, across where it joins the cheek-bone; and, lastly, at its nasal process, parallel with the inferior margins of the lachrymal and nasal bones. I then, with stiong scissors, cut the connexions of the olbitary plate with the os planum of the ethmoid bone, and orbitary process of the palate bone, deep into the orbit, to the spheno-maxillary fissure; and was, lastly, able, by notching with the bone forceps at every point where the saw had been applied, to remove the entire bone, which had its cavity flled with a firm sarcomatous tumour. There were, besides this, two or three soft gelatinous polypi hanging from the mucous membrane of the ethmoid bone, which required to be removed. A little lint was then inserted in the vacuity, to afford support to the flap of the cheek, which was approximated by needles and ligatures, three needles being inserted in the integuments of the nose, two in the upper lip, and four in the cheek. The patient was now carried to bed, and had an opiate administered. The wounds of the integuments healed by the adhesive inflammation. My patient was able to walk about her room by the eighth day; went out to take an airing on the thirtieth day, and left Edinburgh for Dumfries on Fiday the fifth instant.

\section{REMARKS.}

This operation will, no doubt, appear to many as very hazardous; but it is not so in reality. When once the hamorrhage is commanded, by securing the internal maxillary artery, it is comparatively easily effected, and not nearly so formidable as the former operation of trephining and cauterising the antrum. From the minute description now given, many will infer, that it is tedious and painful ; but, besides my anxiety to afford a clear and satisfactory representation of the steps adopted, I may mention, that in performing lithotomy, and this operation on the same day last summer in the Royal Infirmasy, the period of entrance of the lithotomy patient into the operating theatre to the exit of the patient, on whom excision of the upper jaw-bone was performed, occupied only twenty minutes, which includes the time necessarily required to clean and arrange the table and floor between botll opierations. On the whole, it seems to me that chis operation is much less formidable, painful, and protracted, than the one usually practised for tumonrs in the antrum maxillare, and surpasses it in holding out a prospect of permanent cure. I may add, as not unimportant, that the disfiguration of the countenance is rendered very trifling, by the preservation of the cheekbone.

Edinburgh, 34, York Place, March 12, 1830 . 